\title{
Revistas Odontológicas de Mayor Impacto: Una Revisión de su Comportamiento en el Journal Citation Reports
}

\author{
The Highest Impact Dentistry Journals: A Review of their \\ Performance in the Journal Citation Reports
}

\author{
Jesús Alberto Luengo Fereira1*; Cristal Yurixie Díaz Rosas"; Minerva Anaya Alvarez² \& Cesar Iván Gaitán Fonseca ${ }^{3}$
}

LUENGO, F. J. A.; DÍAZ, R. C. Y.; ANAYA, A. M. \& GAITÁN, F. C. I. Revistas odontológicas de mayor impacto: Una revisión de su comportamiento en el Journal Citation Reports. Int. J.Odontostomat., 11(3):287-293, 2017.

RESUMEN: El objetivo del este estudio fue identificar, describir y analizar las revistas odontológicas indexadas en el Journal of Citation Report. Estudio observacional, descriptivo de cohorte transversal sobre las revistas incluidas en la categoría "Dentistry, Oral Surgery \& Medicine" del Journal Citation Reports, edición 2015, realizada durante el periodo Octubre Noviembre 2016. Las variables incluidas en el estudio fueron: ranking de las revistas, titulo, factor de impacto, área temática, país de procedencia, editorial, número de artículos y de citas durante el último año, eigenfactor score y article influence score. Se empleó estadística descriptiva para su análisis. Fueron incluidas 91 revistas en el Journal Citation Reports en su última versión disponible. Periodontology 2000 es la revista con mejor ranking y factor de impacto 4,949. Odontología General es el área temática con mayor número de registros con 22. Estados Unidos posee 31 títulos de revistas. Wiley-Blackwell es la casa editorial más representativa con 24 revistas. Se publicaron 376 artículos en el Journal of Oral Maxilofacial Surgery, mientras que el Journal of Dental Research recibió 16.401 citas. Es necesario que los odontólogos conozcan las características de las revistas incluidas en el Journal Citation Reports con el propósito de realizar una adecuada selección al momento de enviar los resultados de sus investigaciones.

PALABRAS CLAVE: Revistas, publicaciones, investigación.

\section{INTRODUCCIÓN}

La Odontología Basada en Evidencia (OBE) trata fundamentalmente de la aplicación clínica de las mejores evidencias científicas basadas en la experiencia del operador y expectativas del paciente (Alarcón et al., 2015). En la actualidad, las mejores evidencias científicas son consideradas las más originales, innovadoras o de reciente creación.

Actualmente, la velocidad de la generación del conocimiento es solamente alcanzada por las miles de revistas indexadas publicadas día a día alrededor del mundo. Ésta forma ha alcanzado tal relevancia en la difusión del conocimiento que se ha convertido en la principal vía de transmisión de resultados de investigaciones en la mayoría de los campos del conocimiento, como la Odontología.
El primer nivel de este concepto, es la búsqueda de la mejor evidencia científica para la mejor toma de decisiones (Feijoo et al., 2014). Lo importante sería tener las herramientas apropiadas para determinar cuál sería la mejor evidencia. La calidad de las revistas se define por el cumplimiento de un conjunto de pautas editoriales, presentación, gestión y contenidos que garantizan el rigor científico de sus publicaciones, y por tanto abonen a su prestigio (Cantín \& Aravena, 2014).

La calidad y repercusión de la actividad científica se mide actualmente con el factor de impacto $(\mathrm{FI})$, el cual expresa una medida de la frecuencia en la cual un artículo de una revista ha sido citado en un periodo de tiempo (Monje, 2014; Oosthuizen \& Fenton, 2014), sirviendo como instrumento para evaluar la importancia

\footnotetext{
${ }^{1}$ Programa de Especialidad en Odontopediatría. Unidad Académica de Odontología. Universidad Autónoma de Zacatecas, México.

2 Programa de Médico Cirujano Dentista. Unidad Académica de Odontología. Universidad Autónoma de Zacatecas, México.

${ }^{3}$ Doctor en Ciencias Odontológicas. Programa de Maestría en Ciencias Biomédicas. Universidad Autónoma de Zacatecas, México
} 
de una revista específica dentro de un mismo campo científico.

El Eigenfactor Score (ES), es otra manera de evaluar las publicaciones, y su diferencia con el FI radica en que el ES da prioridad a las citas provenientes de revistas influyentes. Como consecuencia, estas revistas ejercen mayor influencia en la clasificación de las revistas a las que hacen referencia. EI ES no considera las autocitas, lo que permite una evaluación más objetiva (Fardi et al., 2011; Sillet et al., 2012; Kianifar et al., 2014; Cantín et al., 2015).

El Acceso Abierto (OA, por sus siglas en inglés), se refiere a la disponibilidad gratuita de información científica en Internet, donde cualquier usuario la pueda leer, descargar, copiar, distribuir, imprimir y usarlos como datos para software, o utilizarlos para cualquier otro propósito legal, sin barreras financieras, legales o técnicas, distintas a las de la conexión a Internet (Carroll, 2011).

La Web of Science (WoS), antigua Web of Knowledge, ofrece actualmente un interfaz intuitivo y versátil, donde se incluyen varios productos de Clarivate Statistics (anterior Thomson Reuters): Conference Proceedings Citation Index, Current Contents, Journal Citation Reports, Medline, Scholar- One Products, Science Citation Index, Science Citation Index Expanded, Social Sciences Citation Index y Web of Science entre otros. Algunos de estos están creados para satisfacer a diversos usuarios, con acceso a un contenido actualizado, de alta calidad y multidisciplinario; por lo que un producto puede formar parte de otro, como ocurre con WoS, que incluye todas las revistas del Science Citation Index Expanded (SCIE), del Social Sciences Citation Index (SCCl) y del Arts \& Humanities Citation Index (AHCl) (Valderrama Méndez, 2013).

El FI era calculado anualmente por el Institute for ISI Web of Science de las revistas incluidas en el Journal Citation Reports (Chang et al., 2013). Las revistas incluidas en la WoS corresponden a las que hace unos años estaban en el listado del ISI, que luego fue expandido a ISI-expanded y que pasara a integrar las bases de datos y productos de Thomson Reuters, actualmente Clarivate Statistics. En áreas específicas, se puede seguir hablando de Science Citation Index Expanded (SCIE), del Social Sciences Citation Index (SCCl), o del Arts \& Humanities Citation Index (AHCl). Estos se encuentran incluidos en WoS, lo que simplifica la terminología al indicar cuales revistas pertenecen a estos importantes índices (Valderrama Méndez). El propósito del presente estudio fue Identificar, describir y analizar las revistas odontológicas de mayor impacto (citadas, indexadas, adjuntas) en el Journal of Citation Report (JCR) Edición 2015.

\section{MATERIAL Y METODO}

Diseño. Estudio observacional, descriptivo y transversal sobre el total de revistas incluidas en la categoría "Dentistry, Oral Surgery \& Medicine" del Journal Citation Reports, durante el periodo Octubre - Noviembre 2016.

Variables. Se consideraron como variables: ranking de las revistas, título, factor de impacto, área temática, país de procedencia, editorial, número de artículos, citas durante el último año, eigenfactor score y article influence score.

Procedimientos e instrumento de recolección de datos. Se realizó una búsqueda electrónica en el Institute for Scientific Information (ISI) Web of Science (WoS), en base a las revistas incluidas en el Journal Citation Reports (edición 2015, publicada en Julio 2016) en la categoría "Dentistry, Oral Surgery \& Medicine". El total de revistas seleccionadas se analizaron conforme al "factor de impacto (FI), área temática, país y editorial". Seguidamente, se determinó el número de revistas según el continente y país de origen. Finalmente, se realizó un análisis de las 10 revistas más importantes sobre: número de artículos, citas durante el último año, eigenfactor score (ES), article influence score (AIS).

Procesamiento estadístico. La información fue almacenada en una base de datos y analizada con el Programa SPSS V-19 (IL, USA), empleando estadística descriptiva con media, desviación estándar, frecuencias y porcentajes.

\section{RESULTADOS}

Se identificaron 91 revistas incluidas en el Journal Citation Reports, en la categoría "Dentistry, Oral Surgery \& Medicine"; observándose una media del FI de 1,598 $\pm 1,024$, siendo las tres principales: Periodontology 2000 $(4,949)$, Journal of Dental Research $(4,602)$, Oral Oncology $(4,286)$. Las áreas temáticas más relevantes fueron Odontología General (22), Cirugía (12), Implantología (9) y Ortodoncia (8). Al considerar las editoriales que respaldan a las principales revistas, 
LUENGO, F. J. A.; DÍAZ, R. C. Y.; ANAYA, A. M. \& GAITÁN, F. C. I. Revistas odontológicas de mayor impacto: Una revisión de su comportamiento en el Journal Citation Reports. Int. J.Odontostomat., 11(3):287-293, 2017.

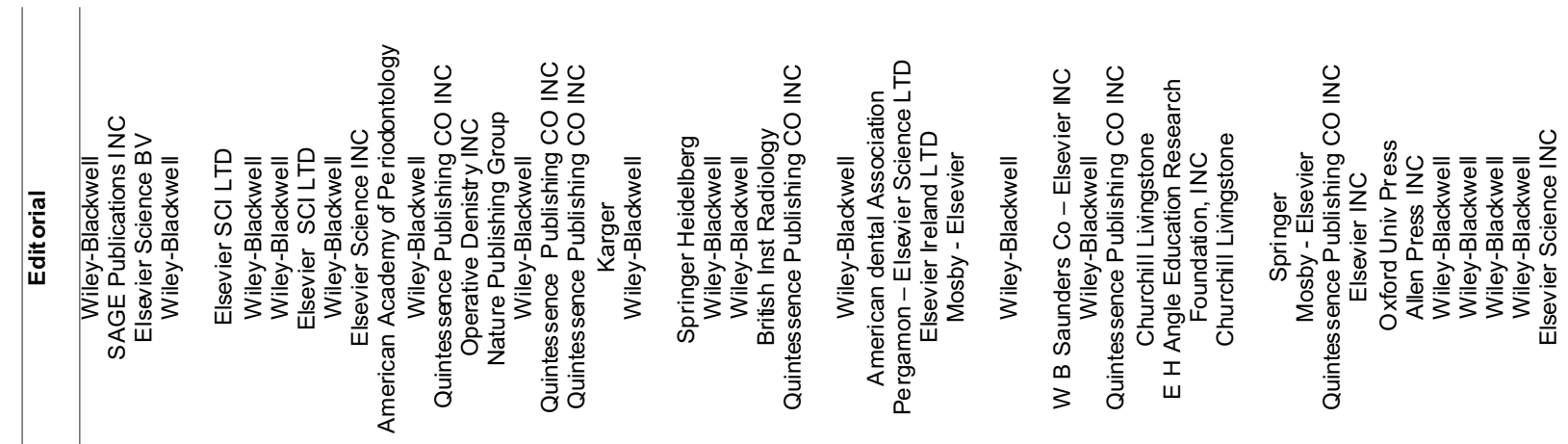

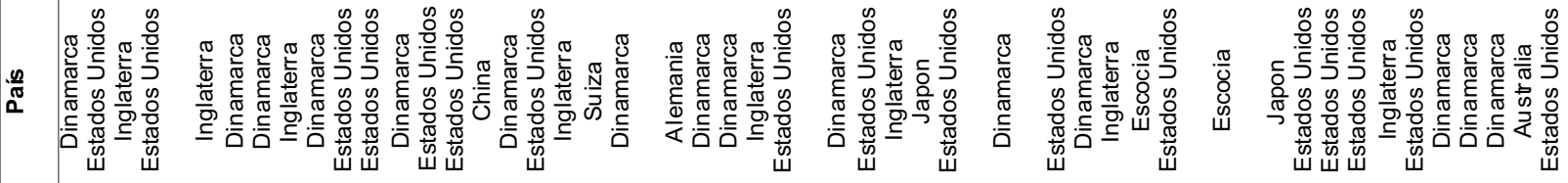

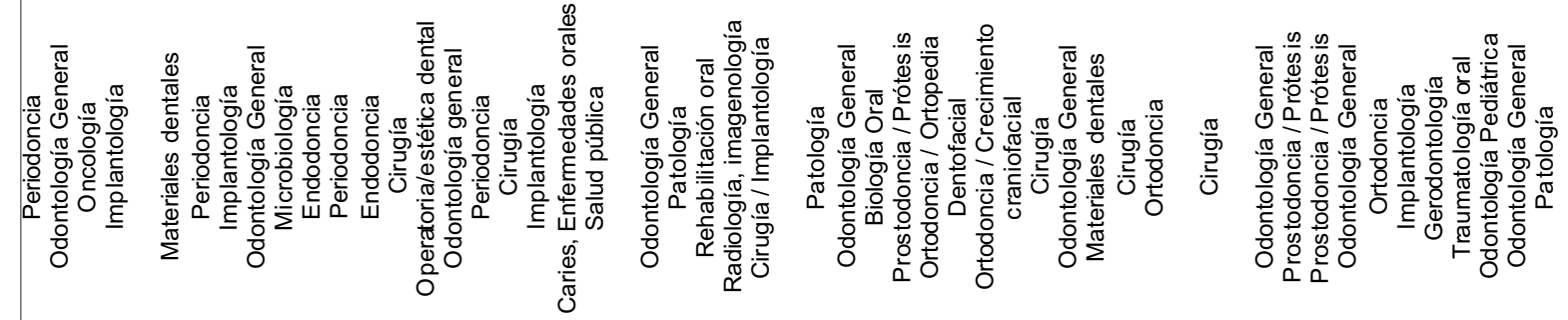

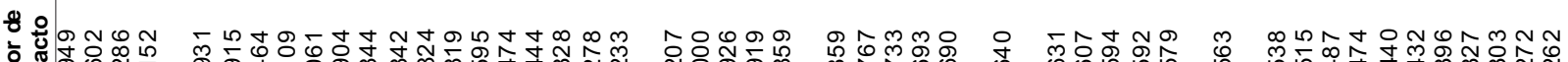
인

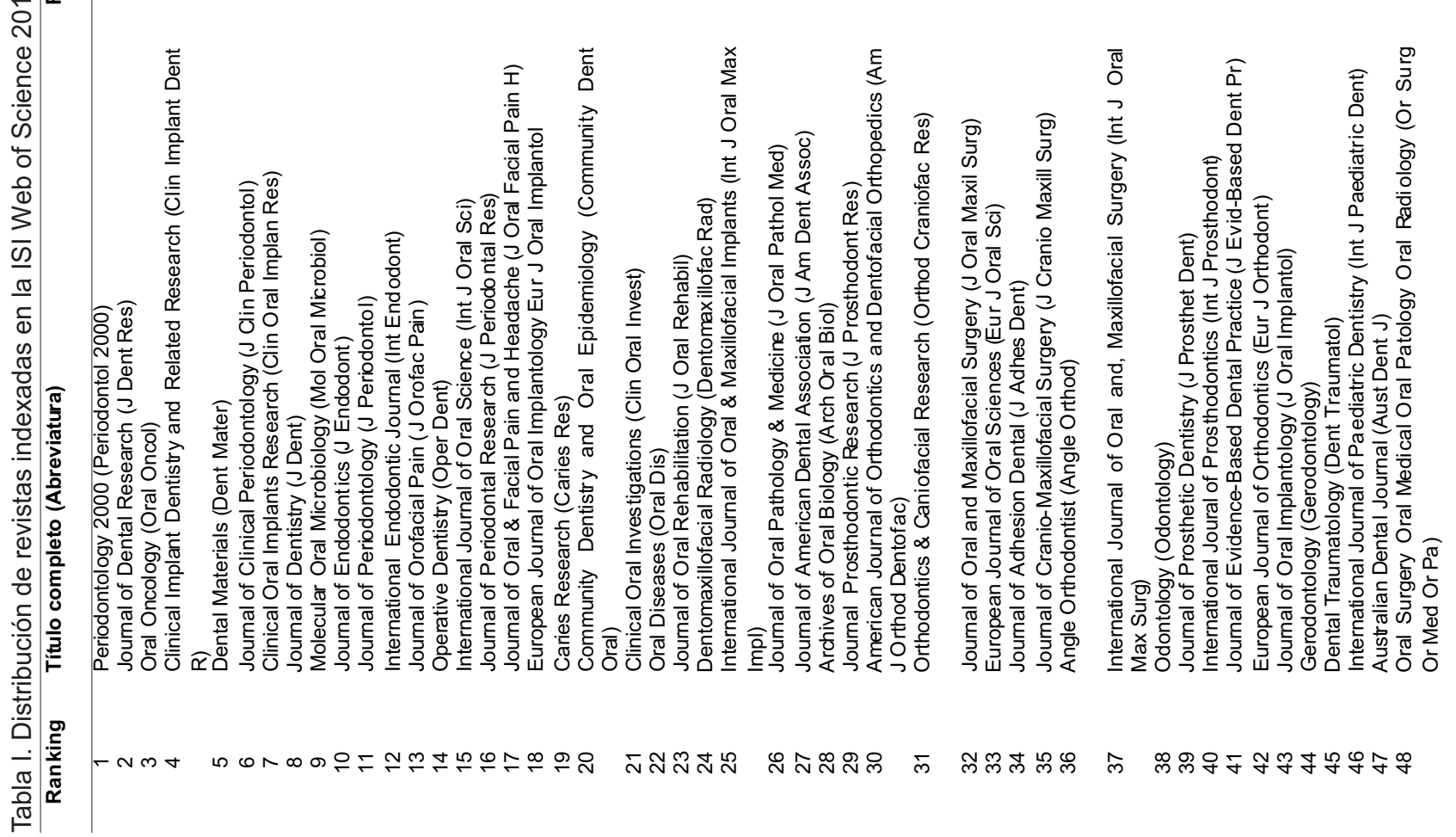



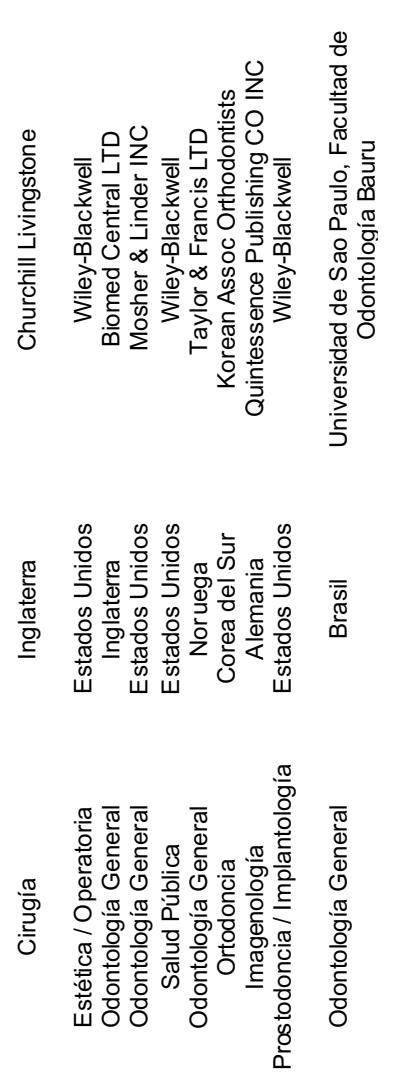

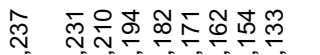

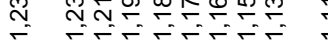

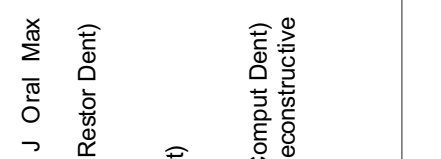

Asia

\begin{tabular}{|c|c|c|c|c|}
\hline \multirow{3}{*}{ Continente } & & \multicolumn{3}{|c|}{ Revistas Científicas } \\
\hline & & \multicolumn{2}{|c|}{ Frecuencia } & \multirow[t]{2}{*}{ MD } \\
\hline & & $\mathrm{n}$ & $\%$ & \\
\hline África & & 0 & 0 & 0 \\
\hline América & & 34 & 37,4 & $11,33 \pm 17,03$ \\
\hline & Brasil & 2 & 5,9 & \\
\hline & Canadá & 1 & 2,9 & \\
\hline & Estados Unidos & 31 & 91,2 & \\
\hline Asia & & 10 & 10,9 & $2,50 \pm 1,91$ \\
\hline & China & 1 & 10 & \\
\hline & Corea del Sur & 3 & 30 & \\
\hline & Japón & 5 & 50 & \\
\hline & Taiwán & 1 & 10 & \\
\hline Europa & & 44 & 48,3 & $4,40 \pm 6,20$ \\
\hline & Alemania & 4 & 9,1 & \\
\hline & Dinamarca & 17 & 38,5 & \\
\hline & Escocia & 2 & 4,5 & \\
\hline & España & 1 & 2,3 & \\
\hline & Francia & 1 & 2,3 & \\
\hline & Inglaterra & 15 & 34,1 & \\
\hline & Italia & 1 & 2,3 & \\
\hline & Noruega & 1 & 2,3 & \\
\hline & Suecia & 1 & 2,3 & \\
\hline & Suiza & 1 & 2,3 & \\
\hline Oceanía & & 3 & 3,4 & 3,00 \\
\hline & Australia & 3 & 100 & \\
\hline TOTAL & & 91 & 100 & \\
\hline
\end{tabular}

destaca Wiley-Blackwell con 24, Elsevier y Quintessence Publishing CO con 10 revistas cada uno (Tabla I).

Al analizar el continente y país de origen, se encontró que casi la mitad están registradas en Europa (44, MD $=4,40 \pm 6,20$ ), siendo Dinamarca e Inglaterra las de mayor cantidad, 17 y 15 respectivamente. No obstante, de manera general Estados Unidos posee mayor cantidad de revistas con 31 (Tabla II).

Conforme al número artículos publicados durante el último año, se obtuvieron 9015 manuscritos, con una media de 99,07 $\pm 78,63$, donde el Journal of Oral Maxillofacial Surgery (376), Journal of Endodontics (335) y Journal of Cranio-Maxillo-Facial Surgery (333), fueron los tres con mayor cantidad de publicaciones (Fig. 1). Respecto al número de citas que recibieron, se contabilizaron 281.803 con una media general de $3096,7 \pm$ 3700,44 , siendo las más importantes Journal of Dental Research (16.401), Journal of Periodontology (14.268), Journal of Oral Maxillofacial Surgery (13.258) (Fig. 2).

Teniendo en cuenta el ES, se obtuvo una media general de 0,00436 $\pm 0,00468$. La Figura 3, muestra las diez revistas con mayor ES, donde destacan Journal of Dental Research $(0,02345)$, Clinical Oral Implants Research $(0,01695)$, Journal of Oral Maxillofacial Surgery $(0,01693)$, dentro de los tres primeros lugares. Considerando el AIS, encontramos una media general de 0,5485 $\pm 0,3144$. En la Figura 4, se observa al Journal of 
LUENGO, F. J. A.; DÍAZ, R. C. Y.; ANAYA, A. M. \& GAITÁN, F. C. I. Revistas odontológicas de mayor impacto: Una revisión de su comportamiento en el Journal Citation Reports. Int. J.Odontostomat., 11(3):287-293, 2017.

Dental Research (1.541), Periodontology 2000 (1.503) y Clinical Implant Dentistry and Related Research
(1.301), ocupando el primer, segundo y tercer lugar respectivamente de las diez revistas con mayor AIS.

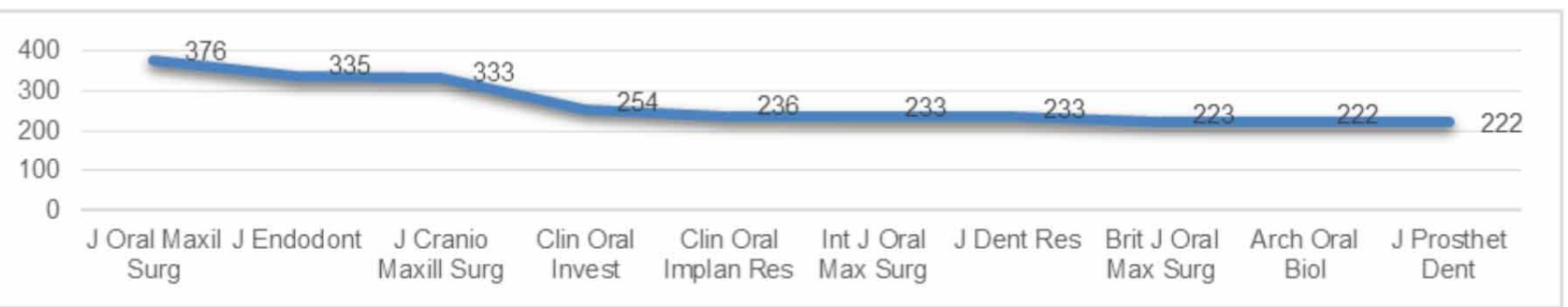

Fig. 1. Top Ten de revistas indexadas en la ISI Web of Science 2015 con mayor número de artículos publicados.

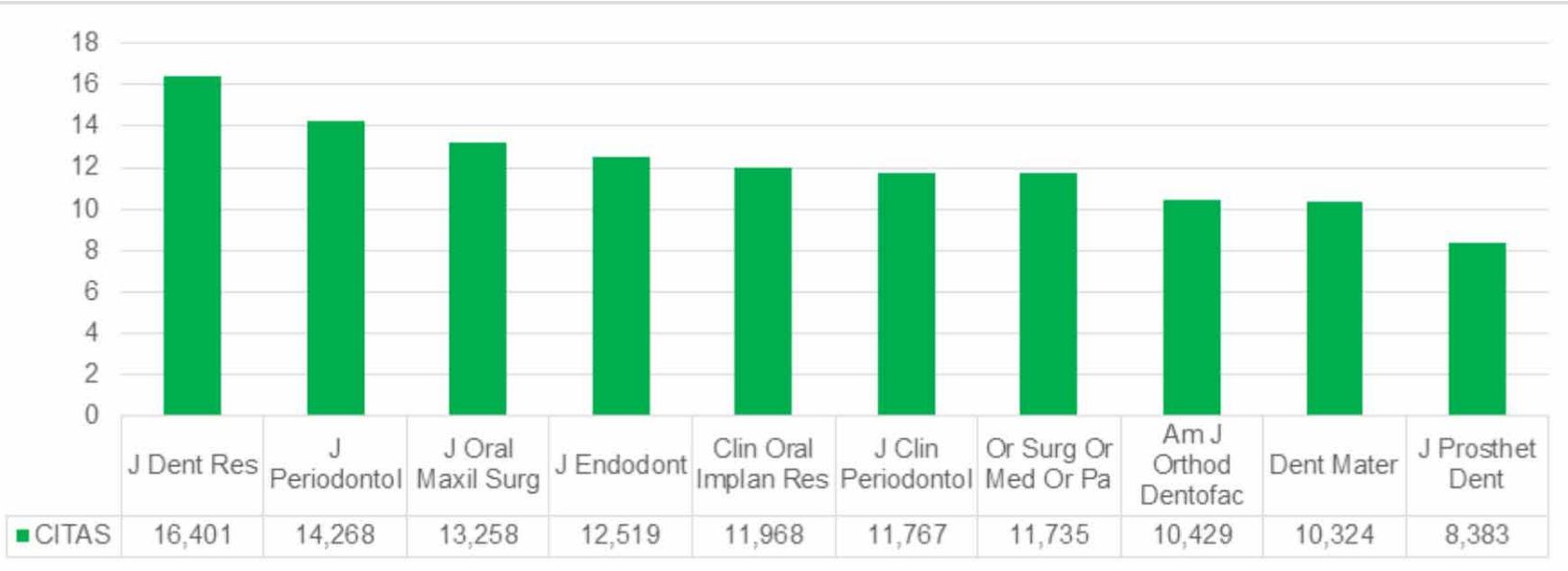

Fig. 2. Top Ten de revistas indexadas en la ISI Web of Science 2015 con mayor número de citas.

\section{EIGENFACTOR SCORE}

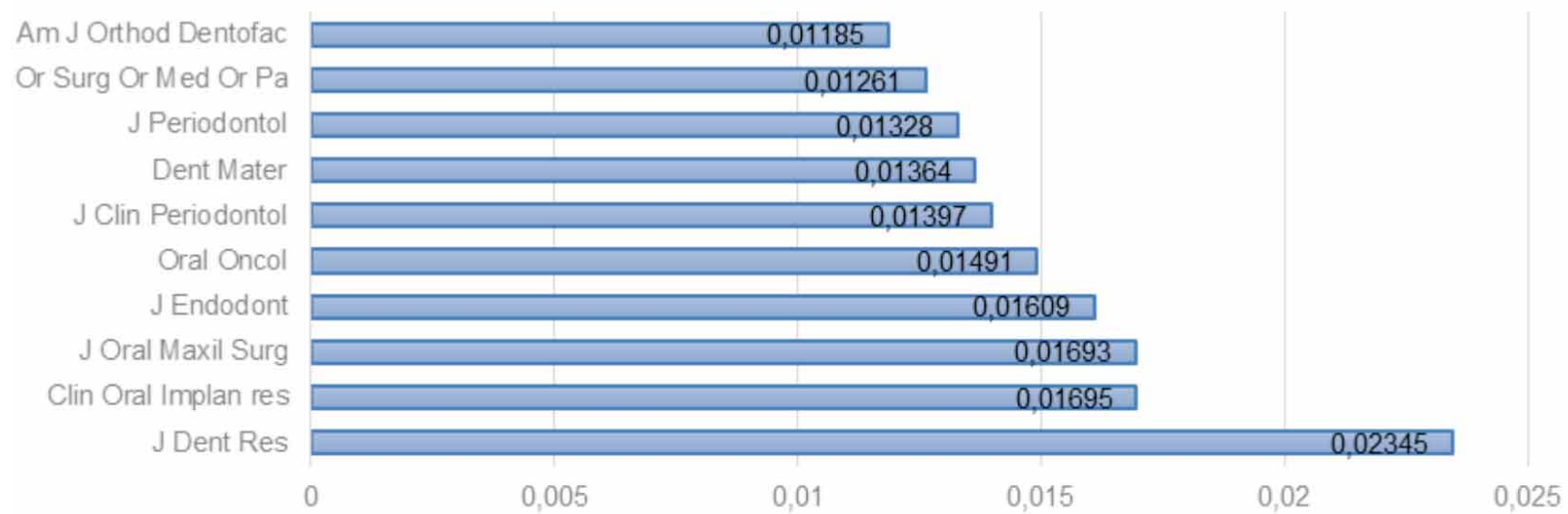

Fig. 3. Top Ten de revistas indexadas en la ISI Web of Science 2015 con mayor Eigenfactor score. 


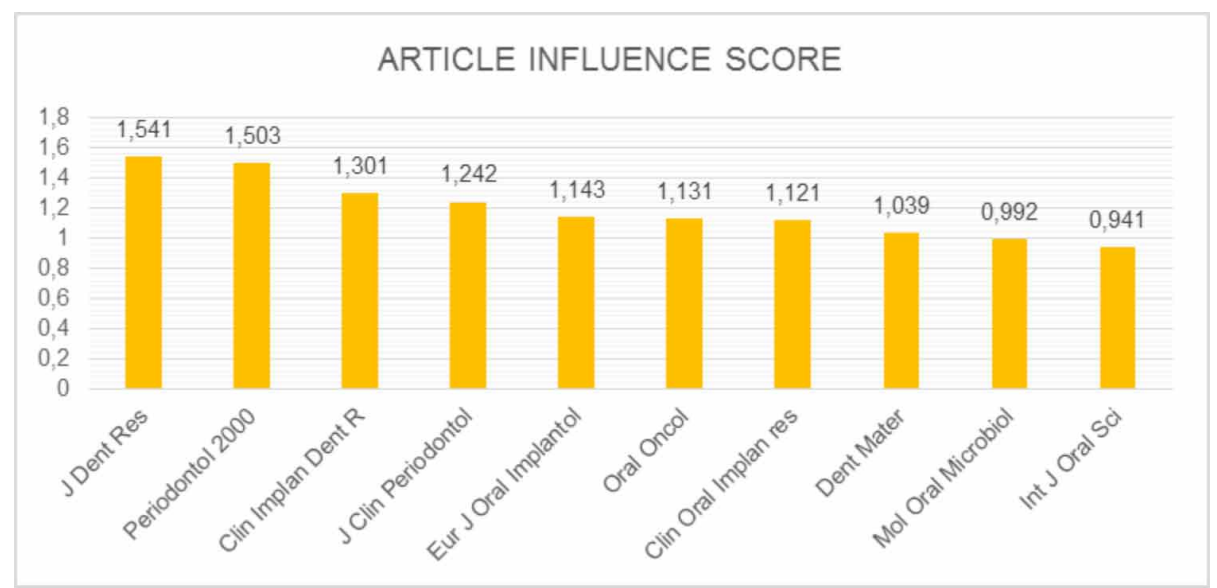

Fig. 4. Top Ten de revistas indexadas en la ISI Web of Science 2015 con mayor Article Influence Score.

\section{DISCUSION}

Las revistas odontológicas incluidas en la categoría "Dentistry, Oral Surgery \& Medicine" del Journal Citation Reports, han venido integrándose e incrementándose de forma exitosa en sus diferentes ediciones anuales, lo que ha favorecido el aumento de publicaciones de ediciones completas en formato electrónico (OA), la producción de indicadores estadísticos y el crecimiento del impacto de la literatura científica en el área de la salud oral. Lo anterior ha sido reflejo de criterios de evaluación con altos estándares de calidad y a la evolución de publicaciones profesionales basadas en la mejor evidencia disponible (Cantín et al.).

En este sentido, los complejos procesos de evaluación por el que pasan las revistas, les ha permitido incorporarse a importantes bases de datos (WoS, Thomson Reuters, SciELO, Citation Index, Scopus, DOAJ), diseñadas para aumentar la visibilidad de sus publicaciones, acceso y difusión; lo que se traduce en un incremento de visitas y de citas sobre los artículos publicados en ellas, y un mayor interés de los investigadores que consultan esas revistas (Corrales et al., 2016).

Una de las principales limitantes para el desarrollo del trabajo fue la poca cantidad de estudios similares disponibles que mostraran una visión actualizada sobre las características de las revistas incluidas en el JCR. No obstante, la evidencia muestra la existencia de una importante cantidad de publicaciones sobre el comportamiento bibliométrico de algunas revistas, incluidas en ciertas bases como WoS,
Clarivate Statistics (Thomson Reuters), JCR, Scielo y Scopus (Cantín et al.; Muthamilarasan \& Prasad, 2014; Alarcón et al.; Corrales et al.).

Por otra parte, aunque se identificaron 91 revistas en la base del JCR, solo 18 países que integran la red poseen revistas en el área, principalmente Estados Unidos (31), Dinamarca (17) e Inglaterra (15). Este predominio de países donde se maneja el idioma en inglés, ha favorecido que sea precisamente éste idioma el idóneo para publicar, gracias a su universalidad, trayendo consigo una mayor visibilidad, lectura y citas. Dicha evidencia demuestra un incremento significativo en el número de revistas incluidas en la categoría "Dentistry, Oral Surgery \& Medicine" del JCR, en comparación de lo encontrado por Alarcón et al.; quienes reportaron 82 revistas incluidas en la edición del 2014. De igual forma, debemos resaltar la calidad científica de la editorial americana Willey Blackwell la cual domina con 24 revistas en su colección, manteniéndose así en las ediciones 2014 y 2015, dentro de las más reconocidas casas editoriales.

Al evaluar el área temática, pudo constatarse una amplia diversidad que se distribuyen entre la odontología general, cirugía, ortodoncia e implantología como las más numerosas; evidenciándose cierta diferencia con respecto al predominio de la temática según la edición anterior (Alarcón et al.), donde figuraban áreas relacionadas con materiales dentales y periodoncia. No obstante y conforme a los avances dentro de la odontología estos temas pueden considerarse como los más relevantes en la actualidad. 
Al analizar el FI, el número de artículos publicados, número de citas recibidas y ES, pudo observarse diferencias entre las revistas, ya que Periodontology 2000 fue la revista con mayor factor de impacto, pero no así en las demás categorías; no obstante, el Journal of Dental Research logró posicionarse con el segundo lugar en $\mathrm{Fl}$ y primer lugar en cuanto a citas recibidas y ES. Esta variación puede deberse a que el índice ES busca ser más preciso en los valores que arroja, debido a que excluye las auto-citas y considera como referencia primordial las citas procedentes de las revistas más influyentes (Muthamilarasan \& Prasad).

Respecto a las citas, estas se han utilizado como un indicador del impacto científico de los artículos y, en muchos campos, la base de la recompensa profesional. Desde 2004, diversos estudios (de diferentes campos científicos) han informado que los artículos de OA se citan significativamente más que los artículos que no son de OA. Sin embargo, revisiones recientes señalan que estos estudios carecían de rigor metodológico (por ejemplo, falta de control de las variables) y que la "ventaja de la cita" que encontraron apareció como resultados de análisis inadecuados (Hua et al., 2016).

Finalmente, es necesario que los odontólogos conozcan las revistas disponibles en JCR y sus características con el fin de hacer una adecuada selección al momento de enviar sus trabajos o investigaciones. Este conocimiento toma mayor relevancia cuando se involucran instituciones, universidades o países, ya que actualmente, parámetros como la investigación científica son parte de los análisis de desarrollo o aportes financieros para ellos, como también un parámetro para obtener recursos (postulación a proyectos), postular a becas o generar productos de proyectos con financiamiento, así como tesis de pre y postgrado.

LUENGO, F. J. A.; DÍAZ, R. C. Y.; ANAYA, A. M. \& GAITÁN, F. C. I. The highest impact dentistry journals: A review of their performance in the Journal Citation Reports. Int. J. Odontostomat., 11(2):287-293, 2017.

ABSTRACT: The objective of this study was to identify, describe and analyze the dental journals indexed in the Journal Citation Reports. Observational, cross - sectional descriptive study on the journals included in the "Dentistry, Oral Surgery \& Medicine" category of the Journal Citation Reports, 2015 edition, during the period October - November 2016. The variables in the study were: ranking of journals, title, impact factor, subject area, country of origin, editorial, number of articles and citations during the last year, eigenfactor score and article influence score. Descriptive statistics were used for analysis. In its latest available version 91 journals were included in the Journal Citation Reports. Periodontology 2000 is the journal with the best ranking and impact factor 4.949. General Dentistry is the subject area with the highest number of records with 22. The United States has 31 journal titles. Wiley-Blackwell is the most representative publishing with 24 journals. There were 376 articles published in the Journal of Oral Maxillofacial Surgery, while the Journal of Dental Research received 16,401 citations. It is necessary that dentists to know the characteristics of the journals included in the Journal Citation Reports in order to make an appropriate selection when sending the results of their research.

\section{KEY WORDS: journals, publications, research.}

\section{REFERENCIAS BIBLIOGRÁFICAS}

Alarcón, M.; Aquino, C.; Quintanilla, C.; Raymundo, L. \& Álvarez, J. Evidence based dentistry: The 82 highest impact journals. Int. J. Odontostomat., 9(1):43-52, 2015.

Cantín, M. \& Aravena, Y. Dental journals in the SciELO database: A bibliometric overview. Int. J. Odontostomat., 8(2):215-20, 2014.

Cantín, M.; Muñoz, M. \& Roa, I. Comparison between Impact Factor, Eigenfactor Score, and SCImago Journal Rank Indicator in anatomy and morphology journals. Int. J. Morphol., 33(3):1183-8, 2015.

Carroll, M. W. Why full open access matters. PLoS Biol., 9(11):e1001210, 2011.

Chang, C. L.; McAleer, M. \& Oxley, L. Coercive journal self citations, impact factor, journal influence and article influence. Math. Comput. Simul., 93:190-7, 2013.

Corrales, I. E.; Reyes, J. J. \& Fornaris, Y. Bibliometric analysis of the Journal of Oral Research. Period 2012-2015. J. Oral Res., 5(5):18893, 2016.

Fardi, A.; Kodonas, K.; Gogos, C. \& Economides, N. Top-cited articles in endodontic journals. J. Endod., 37(9):1183-90, 2011.

Feijoo, J. F.; Limeres, J.; Fernández-Varela, M.; Ramos, I. \& Diz, P. The 100 most cited articles in dentistry. Clin. Oral Investig., 18(3):699706, 2014.

Hua, F.; Sun, H.; Walsh, T.; Worthington, H. \& Glenny, A. M. Open access to journal articles in dentistry: Prevalence and citation impact. J. Dent., 47:41-8, 2016.

Kianifar, H.; Sadeghi, R. \& Zarifmahmoudi, L. Comparison between Impact Factor, Eigenfactor Metrics, and SCimago Journal Rank Indicator of pediatric neurology journals. Acta Inform. Med., 22(2):1036, 2014.

Muthamilarasan, M. \& Prasad, M. Impact of impact factor in quantifying the quality of scientific research. Curr. Sci., 107(8):1233-4, 2014.

Oosthuizen, J. C. \& Fenton, J. E. Alternatives to the impact factor. Surgeon, 12(5):239-43, 2014.

Sillet, A.; Katsahian, S.; Rangé, H.; Czernichow, S. \& Bouchard, P. The Eigenfactor ${ }^{\mathrm{TM}}$ Score in highly specific medical fields: the dental model. J. Dent. Res., 91(4):329-33, 2012.

Valderrama Méndez, J. O. Ya no hay Revistas ISI, sólo revista WoS. Inf. Tecnol., 24(5):1, 2013.

Dirección de correspondencia:

Jesús Alberto Luengo Fereira

Programa de Especialidad en Odontopediatría

Unidad Académica de Odontología

Universidad Autónoma de Zacatecas (UAZ)

Carretera a la Bufa S/N, Colonia Centro Zacatecas,

C.P. 98000

Zacatecas - MÉXICO

Recibido : 11-04-2017

Aceptado: 12-06-2017

E-mail: jluengo@uaz.edu.mx 